\section{PSICOLOGIA IBEROAMERICANA}

\section{Psicología Iberoamericana}

ISSN: 1405-0943

psicología.iberoamericana@uia.mx

Universidad Iberoamericana, Ciudad de

México

México

Orantes López, Claudia; Ayala Guerrero, Fructuoso; Méndez Ubach, Milagros; Oropeza Tena,

Roberto; Juárez García, Francisco L.

Arquitectura de sueño en dependientes de la cocaína en abstinencia crónica

Psicología Iberoamericana, vol. 21, núm. 2, julio-diciembre, 2013, pp. 48-59

Universidad Iberoamericana, Ciudad de México

Distrito Federal, México

Disponible en: http://www.redalyc.org/articulo.oa?id=133930525006

Cómo citar el artículo

- Número completo

- Más información del artículo

- Página de la revista en redalyc.org

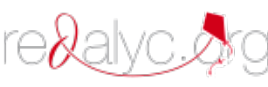

Sistema de Información Científica

Red de Revistas Científicas de América Latina, el Caribe, España y Portugal

Proyecto académico sin fines de lucro, desarrollado bajo la iniciativa de acceso abierto 


\title{
Arquitectura de sueño en dependientes de la cocaína en abstinencia crónica
}

\author{
Sleep Architecture for cocaine addicts during chronic abstinence
}

\author{
Claudia Orantes López ${ }^{1}$ \\ Fructuoso Ayala Guerrero \\ Universidad Nacional Autónoma de México \\ Milagros Méndez Ubach \\ instituto Nacional de Psiquiatría Ramón de la Fuente Muñiz \\ Roberto Oropeza Tena \\ Universidad Michoacana de San Nicolás de Hidalgo \\ Francisco L. Juárez García \\ instituto Nacional de Psiquiatría Ramón de la Fuente Muñiz
}

\section{RESUMEN}

Durante la abstinencia, los consumidores de cocaína tienen problemas de sueño durante al menos tres semanas, relacionados con la frecuencia, la cantidad y el tiempo de consumo. Existe relativamente poca información de las características del sueño en periodos largos de abstinencia a cocaína, ya que no hay reportes después de los tres meses. El objetivo fue estudiar los efectos sobre el sueño en dependientes de la cocaína en abstinencia crónica del tercero al sexto mes. Se realizaron estudios polisomnográficos durante la abstinencia en diez dependientes de la cocaína pareados en sexo, edad e índice de masa corporal (IMC) con diez participantes no consumidores. Los resultados muestran alteraciones en la arquitectura de sueño, disminución en la latencia al sueño y cantidad de sueño mor. Además, hubo aumento significativo en la duración de la fase N1 y en la eficiencia al sueño.

Palabras clave: sueño de ondas lentas sol, sueño de movimientos oculares rápidos MOR, abstinencia crónica, cocaína, polisomnografía.

\begin{abstract}
During abstinence, cocaine users experience sleeping problems for at least three weeks depending on the frequency, amount and length of their habit. Relatively little information exists on the types of sleep during long periods of abstinence from cocaine, since there are no reports after three months of abstinence. The objective of the study was to study the effects on sleep on cocaine addicts during chronic abstinence, from the third to the sixth month. During their abstinence period, ten cocaine addicts were given polysomnographies, by sex, age and body mass index (BMI), and paired with 10 non-users. The results show alterations in sleep architecture, a reduction in sleep latency, and the amount of REM sleep. Additionally, there was a significant increase in the length of the N1 phase and sleep efficiency.

Keywords: slow-wave sleep, rapid eye movement (REM) sleep, chronic abstinence, cocaine, polysomnography.
\end{abstract}

\footnotetext{
${ }^{1}$ Recibido: 26 de diciembre, 2012 - Aceptado: 30 de mayo, 2013

Nota de autor: Los autores de esta investigación no tienen ningún conflicto de intereses. Es parte de la obtención de grado doctoral de Claudia Orantes López en la Universidad Nacional Autónoma de México (UNAM). Becaria (núm. 229160) del Consejo Nacional de Ciencia y Tecnología (Conacyt). Agradecimientos: A la casa de recuperación de alcoholismo y drogadicción Carrasco A. C., por su apoyo en el desarrollo de la investigación. A Néstor Hernández y a Isis García por la colaboración prestada, así como a los colaboradores que apoyaron en la realización de las polisomnografías: Tania García V., Gabriela Vázquez F., David A. Herrera C., Alán García J., Itzel de Aquino H., Alfredo Anguiano S., Lya Resendis Q., y J. Emanuel Molinero H.
} 


\section{INTRODUCCIÓN}

El sueño es un proceso fisiológico constituido por una sucesión de fases que se distribuyen, usualmente, durante el periodo nocturno del ciclo nictameral (Ayala \& Mexicano, 2010a). Se diferencia en sueño sin movimientos oculares rápidos (NMOR) y sueño de movimientos oculares rápidos (MOR). En el sueño NMOR se distinguen tres fases, la N1, la N2, que reciben el nombre de sueño superficial y la N3, que conforma el sueño de ondas lentas (SOL) (Iber, Ancoli-Israel, Chesson \& Quan, 2007).

Los cambios entre la vigilia y el sueño, así como la sucesión de las fases son modulados por conjuntos de neuronas situados en diferentes zonas encefálicas. En estos cambios intervienen agentes químicos y neurotransmisores, como la acetilcolina, la serotonina, la dopamina, entre otros, que facilitan la comunicación entre las neuronas (Ayala \& Mexicano, 2010b).

El sueño es muy sensible a múltiples factores tanto intrínsecos como extrínsecos, de tal manera que se puede afectar su duración y calidad de manera significativa (Prospéro, Guzmán, Méndez, Herrera \& Ruiz, 2009). Dada la importancia que representan para la salud del individuo, los trastornos del sueño se han ordenado dando origen a clasificaciones.

En la última Clasificación Internacional de Trastornos de Sueño se describen ocho categorías, dentro de las cuales se encuentran los trastornos del sueño inducidos por consumo de sustancias. Debido al uso y al abuso de fármacos y drogas, pueden surgir diferentes trastornos del sueño (AASM, 2005). Aquéllos como el insomnio inducido por sustancias alcanza $0.2 \%$ en la población general (Gállego, Toledo, Iriarte \& Urrestarazu, 2007).

Existen numerosos reportes acerca de los efectos producidos por la administración de diferentes sustancias psicoactivas sobre los estados de vigilancia. Las anfetaminas y la cocaína bloquean la recaptura de dopamina (DA), ocasionando un incremento de su concentración en el espacio sináptico, induciendo vigilia sostenida y ejerciendo su acción en el tronco encefálico, el área tegmental ventral, el núcleo accumbens y el sistema mesolímbico, los cuales conforman la vía de recompensa cerebral. En general, las sustancias que incrementan la DA cerebral producen activación y vigilia (Hyde, Roehrs \& Roth, 2005). Por otra parte, varias drogas psicoactivas causan trastornos fisiológicos y cognitivos durante el periodo de abstinencia (Walsh, Stoops, Moody, Shen-Nan Lin \& Bigelow, 2009).

Se ha descrito un cuadro sintomático producido porla abstinencia en sujetos adictosa la cocaína sin otras patologías psiquiátricas (Llopis, 2001). Este cuadro se desarrollaba en tres fases que iniciaban en el momento que se interrumpía el consumo. Con base en estos hallazgos se propuso un modelo trifásico integrado por las fases crash (choque), supresión y extinción. Esta última puede llegar a consolidarse como abstinencia crónica completa o terminar en una recaída.

Weddington et al. (1990) evaluaron cambios en el estado de ánimo, el nivel de deseo de consumo (craving) y la calidad subjetiva de sueño en dependientes de la cocaína, en un periodo de veintiocho días de abstinencia. Observaron síntomas psiquiátricos asociados con el consumo, la disminución del nivel de craving al pasar los días y la dificultad para conciliar el sueño, así como múltiples despertares. Estos autores no encontraron la abstinencia trifásica reportada por Gawin y Kleber (Llopis, 2001; Johanson, Roehrs \& Schuh, 1999).

Satel et al. (1991) evaluaron síntomas de abstinencia a la cocaína durante los primeros veintiún días en personas dependientes, obteniendo resultados similares a los de Weddington (1990), tales como la presencia de síntomas ansioso-depresivos relacionados con el nivel de craving.

Cottler, Shillington, Compton, Mager y Spitznagel (1993) examinaron la sintomatología seguida del cese del consumo, observando que los síntomas dominantes eran depresión, ansiedad, irritabilidad, cansancio, somnolencia, dificultad para iniciar a dormir, problemas de concentración, de alimentación y pulso cardiaco elevado. La intensidad de los síntomas disminuía después de las primeras semanas de abstinencia.

Coffey, Dansky, Carrigan y Brady (2000) también observaron una disminución en la sintomatología presentada en los dependientes a medida que avanzaba el periodo de abstinencia. Evaluaron los síntomas presentes en los primeros veintiocho días divididos en tres bloques de acuerdo con el tiempo de la misma. En relación con la evaluación subjetiva del sueño no hubo cambios en los tres bloques.

Las alteraciones de sueño descritas en dependientes de la cocaína, en fase temprana de abstinencia, se 
han obtenido al evaluar la percepción subjetiva de diferentes aspectos de sueño, o de forma objetiva por medio de estudios polisomnográficos (PSG) (Meana \& Barturen, 1993). Con estos últimos se observó que la macroarquitectura de sueño se encuentra afectada en los consumidores de cocaína y se relaciona con la frecuencia, la cantidad y el tiempo de consumo. La polisomnografía revela un desorden considerable que contribuye con un deterioro cognitivo importante (Poling, Kosten \& Sofuoglu, 2007).

Matuskey Pittman, Forselius, Malison \& Morgan (2011) mostraron datos de tres estudios polisomnográficos durante la abstinencia temprana de cocaína, y evaluaron aspectos subjetivos y objetivos del sueño. Observaron disminuciones en el tiempo total, en el porcentaje de MOR, de N1 y N2, así como menor eficiencia; además, encontraron aumento en la latencia a sueño, en la latencia a MOR e incremento ligero en SOL, en dependientes de la cocaína. Éstos mostraron un deterioro general en las medidas objetivas de sueño en las primeras tres semanas, y las medidas subjetivas mejoraron con el incremento de la abstinencia, dando lugar a la evidencia del "insomnio oculto" durante la abstinencia temprana.

Las alteraciones en el sueño por consumo de cocaína en fase de abstinencia temprana persisten durante al menos tres semanas; sin embargo, no hay pruebas suficientes que indiquen el tiempo (Morgan, Pace-Schott, Sahul, Coric, Stickgold \& Malison, 2006; Pace-Schott, Stickgold, Muzur, Wigren, Ward \& Hart, 2005a).

Morgan y Malison, en una revisión de estudios polisomnográficos, evaluaron medidas objetivas de sueño en dependientes de la cocaína. Los resultados de las revisiones mostraron que las alteraciones más severas se presentaban después de los once días de abstinencia y que no se veía mejoría en la tercera semana (Kowatch, Schnoll \& Knisely, 1992; Morgan \& Malison, 2007).

Aunque existen varios reportes polisomnográficos obtenidosduranteperiodoscortosdeabstinencia, actualmente hay escasa información de este tipo de estudios obtenidos durante periodos de abstinencia crónica en dependientes de la cocaína.

Debido a lo anterior, el objetivo de la presente investigación fue estudiar los efectos del consumo crónico de cocaína sobre el sueño, en dependientes de ella, en periodo de abstinencia crónica, a partir de los tres meses y hasta los seis meses de abstinencia. Presentamos datos transversales y longitudinales del tiempo evaluado, en los dependientes de la cocaína pareados con un grupo de no consumidores.

El sueño es primordial en la salud y es necesario prestarle atención en la problemática de las adicciones (Medina-Mora, 2008). El número de recaídas en los dependientes en rehabilitación es mayor en aquellos que presentan problemas de sueño y trastornos duales vigentes (Drake \& Wallach, 2000; Miró, Martínez \& Arriaza, 2006). Además, conocer las alteraciones electroencefalográficas de la macroarquitectura de sueño en dependientes podría ayudar a adecuar nuevas intervenciones para esta población; asimismo, atender las alteraciones del sueño durante la abstinencia podría ser un factor de protección para evitar futuras recaídas (Brady, 1998; Hyde et al., 2005).

\section{MÉTODO}

El método utilizado fue un estudio descriptivo longitudinal, en el cual se evaluaron pacientes que cumplían con diagnóstico de dependencia a cocaína y que estaban en abstinencia crónica.

\section{Participantes}

La muestra quedó integrada por veinte hombres, divididos en dos grupos; el de observación (GO) estuvo formado por diez dependientes de la cocaína con promedio de edad de $28 \pm 5$, diagnosticados de acuerdo con el criterio de dependencia del DSMIV-TR (APA, 2002) (ver tabla 1).

El grupo referencial (GR) estuvo integrado por diez participantes, con un promedio de edad de $27 \pm$ 6 , sin problemas aparentes de sueño de acuerdo con la entrevista clínica, y sin consumo de sustancias, pareados con los dependientes de acuerdo con edad, sexo e índice de masa corporal (IMC).

Los criterios de inclusión para el Go fueron: saber leer, cumplir con el diagnóstico según el DSMIV-TR para dependencia a cocaína y con el rango de edad (veintecuarenta), no presentar dependencia a otra sustancia y tener al menos tres meses de abstinencia. Para el GR consistieron en: saber leer, cumplir con el rango de edad y no tener consumo de sustancias ni alteraciones en el estado de ánimo. Los criterios de exclusión para ambos grupos fueron la presencia de padecimientos 
Tabla 1. Criterios diagnósticos de dependencia de sustancias (DSMIV-TR)

Un patrón desadaptativo de consumo que conlleva malestar o deterioro (físico, psicológico o social) y junto al que

han de darse al menos tres de los siguientes criterios en algún momento de un periodo continuado de 12 meses:

- Tolerancia

- Abstinencia

- La sustancia es consumida en cantidades mayores o más tiempo de lo que se pretendía en un principio.

- Existe un deseo persistente o esfuerzos infructuosos de interrumpir el consumo o controlarlo.

- Se emplea mucho tiempo en actividades relacionadas con la obtención de la sustancia, consumo o recuperación de sus efectos.

- Se da una importante reducción de las actividades cotidianas del sujeto debido a la ingesta de la sustancia.

- Se continúa consumiendo la sustancia a pesar de tener conciencia de sus potenciales riesgos.

médicos, incluidos los trastornos psiquiátricos como trastorno depresivo mayor (TDM), trastorno de ansiedad generalizada (TAG), trastorno bipolar (TBP) y esquizofrenia, consumo de medicamentos que alteren el sueño o el estado de ánimo setenta y dos horas previas a la polisomnografía, o interrupción de la abstinencia detectada por medio de la prueba antidopaje en orina.

\section{Instrumentos}

El psiquiatra adictólogo realizó la historia clínica y aplicó la entrevista neuropsiquiátrica internacional MINI Plus para descartar comorbilidad psiquiátrica. La MINI Plus es una entrevista diagnóstica que explora los principales trastornos psiquiátricos del Eje i del DSM-IV y el CIE-10. Se han realizado estudios de validez y de confiabilidad comparando la MINI con el SCID-P para el DSM-III-R y el CIDI. Los resultados demuestran que la MINI tiene un puntaje de validez y confiabilidad aceptablemente alto. La MINI Plus es una edición más detallada de la MINI (Ferrando, Bobes-García, Soto \& Gilbert, 1998).

Posteriormente, se hizo una entrevista clínica de sueño para evaluar posibles problemas a partir de la percepción de los participantes del GO, y en el caso del GR se aplicó para descartar alguna alteración presente. Se les proporcionó un diario de sueño semanal para evaluar la percepción con respecto a su calidad y se les aplicaron pruebas antidopaje semanalmente para detectar benzoilecgonina (300ng/ml), principal metabolito de la cocaína en orina. El instrumento que se utilizó para los antidopajes fue Humadrug Cocaine, prueba inmunocromatográfica en un paso para la detección cualitativa de cocaína en orina humana; además, se evaluó cualitativamente el posible consumo de otra sustancia, a través de contacto y comunicación directa con familiares y con los padrinos del centro de rehabilitación, al cual continuaban asistiendo todos los días a su grupo AA.

Las polisomnografías (PSG) se realizaron con un equipo Cadwell con software Easy II, en el laboratorio de sueño ubicado dentro del Laboratorio de Neurociencias en la Facultad de Psicología de la UnAM.

\section{Procedimiento}

Como parte de la preselección de la muestra, el psiquiatra adictólogo realizó la historia clínica a los dependientes, evaluando las sustancias de consumo, la sustancia problema y las características del consumo acostumbrado. Además, llevó a cabo la entrevista clínica MINI Plus. Una vez seleccionada la muestra se realizaron las polisomnografías; el Go inició a los tres meses de abstinencia, a partir del cual se programaron los estudios de sueño, se citó a los participantes en el laboratorio de sueño a partir de las 20:00 h para iniciar la preparación, la colocación de los electrodos y la evaluación de las medidas subjetivas; el registro se inició según el horario habitual de cada participante y terminó alrededor de las 8:00 h ( $\pm 1 \mathrm{~h})$ del día siguiente, registrándose un total de siete horas ( $\pm 1 \mathrm{~h}$ ) de sueño, aproximadamente. El montaje utilizado para el electroencefalograma (EEG) se estableció de acuerdo con el sistema internacional 10-20, EEG: FP1, FP2, F3, F4, F7, F8, C3, C4, O1, O2 (Martínez \& Rojas, 1999). Se realizó electrooculograma, electromiograma de mentón y tíbiales; electrocardiograma, esfuerzo respiratorio torácico y abdominal 
(bandas Pro-Tech modelo 1460), ronquido (sensor de ronquido Cadwell), flujo nasal (termo pares Sleepmate modelo 1401) y oxímetro (sensores Nonin, 8000JFW FlexiWrap). Los registros de sueño fueron calificados siguiendo los criterios del Manual de calificación de sueño y eventos asociados de la Academia Americana de Medicina del Sueño (AASM) (Iber et al., 2007).

Se realizaron tres polisomnografías con la habituación respectiva por cada participante del GO. En el GR se llevó a cabo una polisomnografía por cada participante más la habituación. El tiempo entre cada uno de los estudios fue de un mes y medio. A las personas de cualquiera de los dos grupos que presentaron alteraciones de sueño importantes o presencia de eventos clínicos crónicos, se les proporcionaron técnicas de higiene de sueño y se les canalizó al servicio requerido (clínica de sueño). Se dieron de baja cuatro participantes dependientes durante la investigación por salir positiva la prueba antidopaje durante los primeros dos meses, y otros dos dependientes concluyeron el estudio pero reincidieron en el consumo de cocaína antes de la realización de la tercera PSG. Cinco dependientes que participaron en el estudio fueron referidos a la clínica de sueño.

Semanalmente, se aplicó el diario de sueño, con el cual se evaluaron sus horarios habituales, la percepción del tiempo total de sueño, presencia de pesadillas, despertares durante la noche y eventos estresantes relacionados con el sueño deficiente.

En el Go se realizó, cada semana, la aplicación de antidopajes para verificar su abstinencia; si alguno de los participantes resultó positivo se suspendió su evaluación y no continuó participando en el estudio. La medición de la abstinencia por medio de la prueba antidopaje y la evaluación del diario de sueño se realizaron en el laboratorio de sueño.

\section{Análisis estadístico}

Una vez obtenidos todos los estudios polisomnográficos de los grupos, cada uno se calificó y cuantificó mediante un análisis comparativo de la eficiencia, tiempo total, duración de cada fase, duración promedio de la fase MOR, así como del número de fases. Se realizó un análisis estadístico por medio del paquete estadístico SPss para Windows versión 19.0 (2010), con el propósito de detectar diferencias estadísticamente significativas entre las diferentes variables analizadas.
El primer análisis para evaluar las medidas de tendencia central y la medida de distribución fue descriptivo. Posteriormente, éste se dividió en dos partes; primero se analizaron diferencias entre grupos en cada una de las mediciones transversales del Go (dependientes), comparándolas con el GR. Se utilizaron pruebas estadísticas no paramétricas por el tamaño de la muestra y porque algunas variables no cumplían con una distribución normal. Se compararon, de manera transversal, las estimaciones a los tres meses, a los cuatro meses y medio y a los seis con las calificaciones del GR, a fin de evaluar si existían diferencias en la arquitectura de sueño en los diferentes momentos de abstinencia del grupo de observación. Las variables de la macroarquitectura de sueño que se evaluaron transversalmente fueron latencia a sueño, latencia a $\mathrm{N} 1$, a N2 y N3, latencia de SMOR, duración promedio de MOR expresada en minutos, tiempo total de la N1, de N2, N3 y smor, y se utilizó la prueba U de Mann Whitney para la comparación de dos muestras independientes. En el segundo análisis se realizaron comparaciones dentro del grupo de observación en sus diferentes mediciones longitudinales, con el fin de describir si existen cambios en la macroarquitectura de sueño a lo largo del tiempo, en función de la abstinencia. Las variables de la macroarquitectura de sueño evaluadas fueron las mismas que en U de Mann Whitney. Se utilizó la prueba de Friedman para comparar muestras relacionadas.

\section{RESULTADOS}

Se evaluó a diez dependientes de la cocaína en fase de abstinencia crónica a partir de tres meses, con el fin de detectar si existían alteraciones en la arquitectura de sueño. Las características de los participantes se muestran en la tabla 1.

Quienes integraron el grupo de observación eran policonsumidores cumplían con dependencia a cocaína en forma de crack (piedra) como sustancia de impacto y abuso a diferentes sustancias. Como se mostró en la tabla 1, la media de edad fue de $\bar{X}=28$ años, la media de años de consumo fue de $\bar{X}=5.9$, contaban con una dependencia crónica con múltiples recaídas y varios internamientos en su historial. Ninguno de los participantes presentó comorbilidad psiquiátrica, de acuerdo con la historia clínica realizada por el psi- 
quiatra y la entrevista estructurada MINI plus; tampoco estaban bajo ninguna prescripción médica. La frecuencia de consumo semanal fue de $\bar{X}=6.1$ días. De los diez participantes, seis vivían con su pareja y cuatro solos.

Al momento de iniciar la investigación, los dependientes cumplían con tres meses de abstinencia, como ya se comentó, y estaban recién egresados de un centro de rehabilitación en el que permanecieron durante los meses agudos de la abstinencia. Los participantes continuaron asistiendo a lo largo de la investigación a sus pláticas de AA y a sus sesiones terapéuticas dentro del mismo centro.

En el análisis de la prueba U de Mann Whitney se encontraron diferencias estadísticamente significativas $(\mathrm{p}<0.05)$ en valores de la macroarquitectura de sueño. En la tabla 2 se muestran los resultados de los diferentes análisis.

Tabla 1. Características de la muestra

\begin{tabular}{|c|c|c|}
\hline & Grupo observación & Grupo referencial \\
\hline & $N=10$ & $N=10$ \\
\hline $\operatorname{Edad}[\bar{X}(D E)]$ & $28(5)$ & $27(6)$ \\
\hline Sexo & masculino & masculino \\
\hline Sustancias consumidas & policonsumo & alcohol (ocasional) \\
\hline Sustancia problema & crack & ninguna \\
\hline Años de consumo & 5.9 & 0 \\
\hline Frecuencia (semanal) & 6.1 & 0 \\
\hline Con pareja & 0.6 & 0.5 \\
\hline Sin pareja & 0.4 & 0.5 \\
\hline
\end{tabular}

Nota:

Sustancias consumidas: alcohol, tabaco, marihuana, anfetaminas, cocaína en polvo, crack.

Sustancia problema: aquélla a la que son dependientes, crack.

Años de consumo: tiempo de consumo de crack.

Frecuencia: número de veces que había consumo en una semana.

Tabla 2. Resultados de los diferentes análisis estadísticos transversales y longitudinales

\begin{tabular}{|c|c|c|c|c|c|c|c|c|c|}
\hline & \multirow{2}{*}{\multicolumn{2}{|c|}{$\begin{array}{c}\text { Referencial }(\mathrm{N}=10) \\
\text { psg }\end{array}$}} & \multicolumn{6}{|c|}{ Observación ( $\mathrm{N}=8$ ) } & \multirow[b]{3}{*}{ Friedman } \\
\hline & & & \multicolumn{2}{|c|}{ psg1 } & \multicolumn{2}{|c|}{ psg2 } & \multicolumn{2}{|c|}{ psg3 } & \\
\hline & $\bar{x}$ & $\mathrm{DE}$ & $\bar{x}$ & $\mathrm{DE}$ & $\bar{x}$ & $\mathrm{DE}$ & $\bar{x}$ & $\mathrm{DE}$ & \\
\hline Eficiencia & 0.9 & 0.0 & 0.96 & 0.03 & 0.96 & 0.03 & 0.96 & 0.03 & $>0.05$ \\
\hline Lat N1 & 8.7 & 5.4 & $5.0^{*}$ & 2.7 & 6.0 & 4.7 & 5.4 & 4.8 & $>0.05$ \\
\hline Lat N2 & 18.2 & 9.4 & 11.0 & 5.8 & 13.5 & 7.6 & $10.2 *$ & 5.2 & $>0.05$ \\
\hline Lat N3 & 26.8 & 12.3 & 22.5 & 8.2 & 25.4 & 12.1 & 20.8 & 6.7 & $>0.05$ \\
\hline Lat MOR & 111.6 & 31.7 & $87.4^{*}$ & 30.1 & 94.1 & 45.1 & $80.1^{*}$ & 40.1 & $>0.05$ \\
\hline Prom. MOR & 17.1 & 3.3 & 16.8 & 6.0 & 16.5 & 6.1 & 16.5 & 4.5 & $>0.05$ \\
\hline TTN1 & 49.3 & 23.0 & 49.0 & 25.9 & 55.2 & 19.5 & 48.4 & 23.3 & $>0.05$ \\
\hline TTN2 & 189.1 & 34.9 & 194.8 & 36.0 & 184.9 & 29.3 & 179.6 & 38.4 & $>0.05$ \\
\hline TTN3 & 120.4 & 26.7 & 99.6 & 31.6 & $89.2^{*}$ & 19.0 & 92.0 & 36.9 & $>0.05$ \\
\hline TTMOR & 81.7 & 20.8 & 87.9 & 19.4 & 88.8 & 25.8 & 80.3 & 26.7 & $>0.05$ \\
\hline
\end{tabular}

Nota: $\bar{x}:$ media. DE: desviación estándar.

* U de Mann Whitney $(p<0.05)$. Friedman: resultados en el valor de significancia de la prueba Friedman. Lat N1: latencia a fase 1 de sueño. Lat N2: latencia a fase 2 de sueño. Lat N3: latencia a fase 3 de sueño. Lat Mor: latencia a fase Mor. TTN1: tiempo total de la fase 1. TTN2: tiempo total de la fase 2. TTN3: tiempo total de la fase 3. TTMOR: tiempo total de la fase MOR. Prom. MOR: duración promedio de MOR expresada en minutos. La significancia utilizada fue sig. exacta [2* (Sig. unilateral)] debido al tamaño de la muestra. 
En la primera comparación transversal con el GR a los tres meses de abstinencia se evaluó la macroarquitectura de sueño, encontrándose diferencias estadísticamente significativas en algunas variables como los tieMpos de latencias que fueron distintos en la N1 $(\mathrm{z}=-2.015, \mathrm{p}<0.05)$ entre GO $(\overline{\mathrm{X}}=5.0)$ y el GR $(\overline{\mathrm{X}}=$ $8.7)$, y en el sueño MOR $(z=-2.042, p<0.05)$, GO $(\bar{X}=87.4)$ y GR $(\bar{X}=111.6)$.

En la comparación transversal a los cuatro meses y medio de abstinencia se encontraron diferencias en el tiempo total de duración de la N3 $(\mathrm{z}=-2.533, \mathrm{p}<0.05)$ entre GO $(\bar{X}=89.2)$ y el GR $(\bar{X}=120.4)$.

En la última comparación a los seis meses de abstinencia se vieron diferencias en los tiempos de latencias de N2 $(\mathrm{z}=-2.093, \mathrm{p}<0.05)$, GO $(\overline{\mathrm{x}}=10.2)$ y GR $(\overline{\mathrm{x}}=$ $18.2)$, y de MOR $(\mathrm{z}=-2.046, \mathrm{p}<0.05)$, GO $(\bar{x}=80.1)$ y GR $(\bar{X}=111.6)$.

Longitudinalmente, se compararon los diferentes tiempos de abstinencia en el GO con las muestras relacionadas, utilizando la prueba de Friedman; es importante mencionar que en este paso no se comparó con el GR. Se comparó la macroarquitectura a los tres meses, a los cuatro meses y medio y a los seis. Los resultados mostraron que no hubo diferencias estadísticamente significativas en ninguna de las variables de la macroarquitectura de sueño en los dependientes $(p>0.05)$. Con esto no hay evidencia de que las distribuciones de la macroarquitectura en el tiempo, entre los dependientes, sean diferentes.

En las medias de las latencias en los tres registros de sueño dentro del Go se vieron datos muy similares en los diferentes momentos de abstinencia. Al compararlos con los datos del GR se encontraron diferencias estadísticamente significativas en la latencia a N1, con una $\bar{X}=8.7$ del GR contra $\bar{X}=5.4$ (promedio de las tres mediciones del Go). La latencia a N2 mostró una $\bar{X}=$ 18.2 del GR contra $\bar{X}=11.5$ del Go. En la latencia a MOR se vio una $\bar{X}=111.6$ del GR contra $\bar{X}=87.2$ del promedio. En los tiempos totales de las fases hubo diferencias estadísticamente significativas en la N3 de la segunda PSG con una $\bar{X}=89.2$ contra $\bar{X}=120.4$ del GR.

Se analizaron los porcentajes totales de cada fase en los dos grupos. Los resultados se muestran en la tabla 3 . Se observó que el Go tuvo mayor cantidad de N1 que el GR, alcanzando la máxima cantidad en la segunda PSG a los cuatro meses y medio de abstinencia. En las otras fases, el porcentaje se encontró dentro del promedio y se pudo ver que en las tres mediciones a lo largo del tiempo, los porcentajes totales de N1 no se redujeron, lo cual indica que la presencia de latencia corta a sueño continúa después de los tres meses de abstinencia y hasta los seis meses.

Tabla 3. Porcentajes de fases en los grupos evaluados en comparación con una muestra normativa

\begin{tabular}{|c|c|c|c|c|}
\cline { 2 - 5 } \multicolumn{1}{c|}{} & \multicolumn{3}{c|}{ go } & gr \\
\hline & PSG1 & PSG2 & PSG3 & PSG \\
\hline Fases & \% G & \% G & \% G & \% G \\
\hline N1 & 10.5 & 12.4 & 11.8 & 9.7 \\
\hline N2 & 43.5 & 41.9 & 43.4 & 40.2 \\
\hline N3 & 22.4 & 20.6 & 22.6 & 25.3 \\
\hline MOR & 19.5 & 20.1 & 19.5 & 20.3 \\
\hline
\end{tabular}

Nota: Fases: diferentes fases de sueño. N1: fase 1 de sueño. N2: fase 2 de sueño. N3: fase 3 de sueño. MOR: fase de movimientos oculares rápidos de sueño. PSG1-PSG2-PsG3: Polisomnografías en diferentes momentos de abstinencia. PsG: Polisomnografía del grupo referencial. \% G: porcentajes del grupo por cada fase de sueño.

En el diario de sueño se evaluó la percepción de los participantes del Go en relación con su calidad de sueño y cómo se sentían al despertar, por medio de dos escalas de tipo análogo visual de cero a diez. Se evaluó la presencia de despertares durante la noche y el número de horas que durmieron, aproximadamente. Los resultados mostraron diferencias importantes entre los datos de las polisomnografías y la percepción de los participantes, como se muestra en la tabla 4.

Al evaluar el diario de sueño, se encontraron los siguientes datos: el promedio de horas de sueño por noche fue $\bar{X}=7.4$, lo cual nos indica un tiempo dentro de lo adecuado y que no debería ser la causa de la presencia de privación de sueño. El promedio de latencia a sueño fue de $\bar{X}=12.57$, es decir los dependientes tardan aproximadamente doce minutos en quedarse dormidos; esto es diferente a los datos polisomnográficos que indican una media de latencia a sueño de $\bar{X}=5.4$ minutos. El promedio de la percepción a sentirse cansado o descansado después de despertar fue de $\bar{X}=4.97$, encontrándose casi a la mitad 
Tabla 4. Puntuaciones obtenidas de la percepción de los dependientes en el diario de sueño en diferentes variables

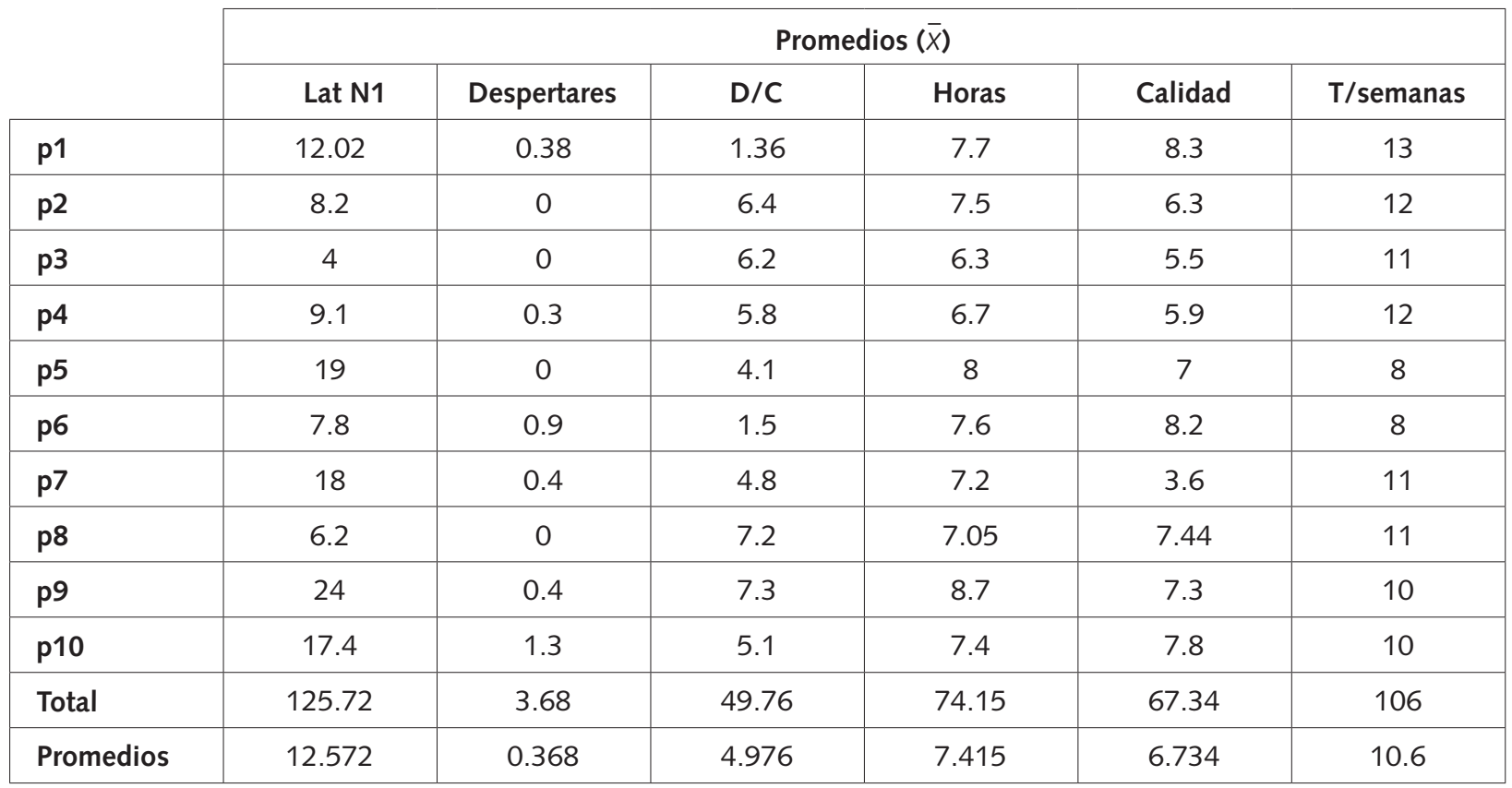

Nota: p1-p2-p3-p... p10: paciente 1, 2, 3, 4...10. LatN1: latencia a fase 1 de sueño. Despertares: número de despertares por noche. D/C: descansado/ cansado, de una escala análoga visual donde cero es descansado y diez es cansado. Horas: número de horas de sueño. Calidad: percepción de la calidad de sueño en una escala análoga visual donde cero es mala calidad y diez buena calidad. T/semanas: tiempo en semanas de cada participante en que se realizó el diario de sueño.

de la escala visual análoga donde $0=$ descansado $10=$ cansado, y coincide con lo reportado por los pacientes al decir que no tienen un sueño reparador, que sienten dificultad para despertar y ponerse activos en el día y se sienten cansados y somnolientos. La media de la calidad de sueño reportada fue de $\bar{X}=6.73$, evaluada con base en una escala análoga visual donde $0=$ mala calidad y 10 = buena calidad, encontrándose un resultado más positivo en su percepción de calidad de sueño. Al comparar la percepción de calidad de sueño con los datos polisomnográficos de la eficiencia, observamos que la eficiencia del sueño también es positiva. Otra manifestación fue tener pesadillas asociadas al consumo, por lo menos durante el primer mes de seguimiento.

\section{DISCUSIÓN}

En las investigaciones realizadas para evaluar la abstinencia aguda a cocaína se han reportado patrones de eficiencia al sueño bajos (Gillin, Pulvirenti, Withers, Golshan \& Koob, 1994; Thompson, Gillin, Irwin \& Golshan, 1995; Morgan \& Malison, 2007; Bracken, Penetar, Rodolico, Ryan \& Scott, 2011). La eficiencia es un término que se utiliza en las mediciones poli- somnográficas y se obtiene de multiplicar el tiempo total de sueño (TTS) por cien y dividirlo por el tiempo pasado en la cama.

Se ha descrito que personas privadas de sueño presentan una mejor eficiencia a éste, es decir, se convierten en durmientes con un sueño más eficiente (De la Calzada, 2007). Esto se explica, en parte, por el hecho de que se presenta con una proporción más alta de ondas delta (N3), que han sido relacionadas con la función de recuperación (Esqueda \& Velázquez, 2011).

Existe el síndrome de sueño insuficiente que se produce en personas que no duermen el tiempo necesario para obtener un nivel de vigilia óptimo durante el día. Hay privación crónica de sueño, y se presenta comúnmente en trabajadores con cambios de turnos o que atraviesan con frecuencia varios husos horarios. El síntoma principal es la excesiva somnolencia diurna principalmente al mediodía, al atardecer o después de las comidas. Duermen de cinco a seis horas los días laborales y unas nueve horas los fines de semana. El despertar matutino es muy dificultoso y con sensación de confusión o de "borrachera de sueño". Les cuesta trabajo realizar tareas diurnas que requieren atención 
y vigilancia sostenida. En estudios polisomnográficos se ha observado una eficiencia del sueño muy elevada $>90 \%$ y una latencia de sueño corta $<10$ minutos (AASM, 2005; De la Calzada, 2007).

Los resultados de esta investigación evidencian que los dependientes de la cocaína mostraron un nivel de eficiencia mejor que los participantes del GR. Esto se puede explicar, en parte, como un efecto compensatorio ya que invirtieron menos tiempo durmiendo, por lo que su latencia para empezar a dormir fue más corta y su sueño fue prácticamente continuo sin interrupciones significativas. Estos hallazgos sugieren que los adictos por lo general no duermen el tiempo necesario, mostrando un síndrome de sueño insuficiente.

Al evaluar los porcentajes de cada fase, se observó que a los tres meses de abstinencia el grupo de dependientes presentó mayor cantidad de N1, en comparación con el GR. Esto indica que hay mayor cantidad de sueño superficial, interrumpido por frecuentes y breves activaciones cerebrales, generando sueño fragmentado. Esto altera la calidad de vida originando somnolencia diurna (SED), fatiga y sensación de malestar personal, así como deterioro del comportamiento social, laboral, problemas de atención, etc. (Castro-Manglano \& Sarrais, 2011).

Conforme pasó más tiempo de abstinencia, la cantidad de N1 aumentó, lo cual indica que el sueño superficial aumenta afectando su calidad. En contraste, el GR manifestó, al inicio y al final de los registros, mayor cantidad de vigilia, mientras que el resto del registro fue ocupado por sueño prácticamente continuo, estando mejor consolidado ya que hubo menos interrupciones. Por el contrario, a pesar de que el Go presentó latencias cortas mostró numerosos cambios de fase, así como sueño fraccionado y activación electroencefalográfica sin producir un despertar conductual. Esto indica que aunque en los porcentajes comparados en cada fase de sueño no hubo diferencias estadísticamente significativas, sí las hubo en cuanto a la calidad del sueño.

A los tres meses el Go mostró una latencia a la N1 estadísticamente significativa, siendo más corta que GR; esto podría deberse a que los dependientes acababan de egresar del centro de rehabilitación donde estaban sometidos a una disciplina estricta; no se les permitía tener siestas diurnas a pesar de sentir la necesidad de dormir, puesto que el periodo que utilizaban era relativamente reducido: se acostaban a las 23:00 h y se levantaban a las 5:00 $\mathrm{h}$.

A los cuatro meses y medio y a los seis meses ya no hubo diferencias significativas entre los dos grupos debidas, probablemente, a que en ese periodo el sueño empieza a recuperarse del efecto previo ocasionado por el consumo crónico de cocaína y por las condiciones en las que estuvieron en recuperación.

La latencia a la N2 fue menor en las tres polisomnografías de los dependientes; sin embargo, sólo la reducción fue estadísticamente significativa en la tercera evaluación realizada a los seis meses, coincidiendo con la reducción de las latencias de todas las fases de sueño y con menos interrupciones por activación electroencefalográfica.

En la latencia a la N3 se presentó una reducción gradual a partir del primer al tercer estudio polisomnográfico; sin embargo, las cifras obtenidas no tuvieron significancia estadística. Por último, las latencias al sueño MOR presentaron reducciones estadísticamente significativas a los tres y a los seis meses. Esto coincide con los reportes de otros autores que han encontrado disminución significativa en un periodo de dos a tres semanas (Morgan, Paliwal, Malison \& Sinha, 2009).

Se ha descrito que la latencia al sueño MOR durante la fase de abstinencia aguda se reduce en comparación con la latencia normal de noventa minutos observada en voluntarios sanos (Pollak, Thorpy \& Yager, 1999). Asimismo, a medida que el tiempo de abstinencia es mayor, la latencia a MOR aumenta, sin llegar al rango normal dentro del tiempo evaluado, pero prediciendo que continuará con la tendencia en esa dirección (Morgan et al., 2009; Bolla et al., 2008). Estos estudios sugieren que este fenómeno podría deberse a un repunte inicial de la supresión de MOR inducida por cocaína.

En los tiempos totales invertidos por el Go en las diferentes fases de sueño sólo se encontraron reducciones significativas en la fase $\mathrm{N} 3$ en el segundo registro, es decir, a los cuatro meses y medio, incrementándose posteriormente en el registro obtenido a los seis meses. Esta tendencia ha sido descrita por otros autores en dependientes al alcohol (Matuskey et al., 2011).

El sol está asociado con un descenso del tono vascular periférico y de otras funciones vegetativas del cuerpo. También se acompaña de una disminución entre $10 \%$ a $30 \%$ de la presión sanguínea, del ritmo 
respiratorio y del metabolismo basal. Es un sueño profundo, de descanso, donde el cuerpo se repone de su desgaste diario, por lo tanto si el sol disminuye, se ven afectadas todas estas funciones (Kovalzon, 1990).

La duración promedio del sueño MOR tuvo una ligera reducción; sin embargo, no alcanzó niveles estadísticamente significativos, quizá por el tamaño de la muestra. La duración promedio de MOR en el GO fue similar en los dos primeros registros, mostrando un ligero incremento en el tercero realizado a los seis meses. Esto coincide con la tendencia seguida por el sol, lo cual indica que en este periodo el sueño tiende a recuperar los niveles normales, como han mencionado otros autores con el consumo de alcohol (Matuskey et al., 2011).

Se ha descrito que en los dependientes de la cocaína, durante la fase de abstinencia aguda, se altera la percepción subjetiva del sueño y que los síntomas más reportados son dificultad para conciliarlo y confusión al despertar (Weddington et al., 1990).

Existen hallazgos paradójicos ya que se ha reportado que las variables subjetivas de sueño mejoran de acuerdo con la percepción del dependiente, mientras que los datos objetivos obtenidos por medio de registros polisomnográficos muestran que el sueño empeora. Esto ha dado lugar a la idea de un "insomnio oculto", una anormalidad funcional del sueño que no es reconocido como tal por la persona que lo experimenta (Morgan et al., 2006).

Matuskey et al. (2011) compararon valores subjetivos y objetivos obtenidos a las tres semanas de abstinencia, observando diferencias significativas sólo en la eficiencia al sueño y en latencia a él. Los resultados de la percepción subjetiva del sueño que obtuvimos en los dependientes por medio del diario coinciden con los resultados de esos estudios. El tiempo promedio subjetivo de la latencia a $\mathrm{N} 1$ fue de 12 minutos, mientras que el promedio objetivo fue de 5.4 minutos, lo cual se parece a lo establecido con el insomnio oculto.

En nuestro estudio, el tiempo subjetivo de sueño no mostró diferencias con el real; sin embargo, se observó que el tiempo de sueño en los dependientes era mayor los fines de semana (11.5 horas) en comparación al promedio obtenido entre semana (7.4 horas). Este patrón es similar al observado en el síndrome de sueño insuficiente ya descrito, donde los horarios de sueño varían y se observa un rebote de sueño los días de descanso.

De acuerdo con Terán (2010), el tiempo de sueño recomendado es entre siete a ocho horas diarias; sin embargo, esto depende de dos factores principales, que son: la necesidad de dormir según nuestras actividades diarias, como parte de la homeostasis, y sentirse con suficiente energía para realizarlas, de no ser así podría existir una alteración en nuestra calidad de sueño. La percepción reportada en relación con la ésta tampoco mostró diferencias significativas entre valores subjetivos y objetivos.

Existen pruebas consistentes de que no hay una disminución en la calidad del sueño, vista desde la percepción de los dependientes a medida que avanza su abstinencia; la calidad del sueño percibida y las medidas subjetivas como la fatiga, el estado de alerta y la concentración mejoran durante las primeras dos a cuatro semanas de abstinencia (Morgan \& Malison, 2007). Los usuarios crónicos de cocaína no reportan problemas en cuanto a que la calidad del sueño en general y la satisfacción con el sueño son comparables con los sujetos control. Se mantienen estables durante la abstinencia debido a la desregulación de la unidad homeostática del sueño (Weddington et al., 1990).

Otras explicaciones son que los consumidores dependientes crónicos de cocaína simplemente necesitan dormir menos, ya que presentan un sueño más reparador (Morgan et al., 2006; Pace-Schott et al., 2005b). Estos hallazgos sugieren que las alteraciones en los consumidores dependientes crónicos son, en realidad, deficiencias que pueden tener como consecuencia funcional un mal apego al tratamiento y propensión a recaídas (Teichner, Horner, Roitzsch, Herron \& Thevos, 2002).

Hubo algunas limitaciones en la investigación como el tamaño pequeño de la muestra, y que la presencia de consumo de otras sustancias, aparte de la sustancia problema (cocaína) durante la investigación, se hizo por medio de técnicas cualitativas como la observación y la comunicación directa. Sin embargo, sería importante que en investigaciones futuras se evaluara una muestra más grande y se aplicaran métodos más precisos que permitieran asegurar el no consumo de sustancias psicoactivas, como por ejemplo el alcohol. • 


\section{REFERENCIAS}

American Academy of Sleep Medicine (AASM). (2005). International classification of sleep disorders, revised: diagnostic and coding manual. Chicago: American Academy of Sleep Medicine.

American Psychiatric Association (APA). Manual diagnóstico y estadístico de los trastornos mentales [DSMIV-TR] (2002). Barcelona: Masson.

Ayala, G. F \& Mexicano, R.M. (2010a). Organización del sueño normal. En Efecto del dolor sobre el sueño (pp. 1-25). México: Mente Abierta:

Ayala, G. F \& Mexicano, R.M. (2010b). Organización del sueño normal. En Efecto del dolor sobre el sueño (pp. 27-45). México: Mente Abierta:

Bolla, K. I., Lesage, S. R., Gamaldo, C. E., Neubauer, D. N., Funderburk, F. R., Cadet, J. L., David, P. M., VerdejoGarcia, A. \& Benbrook, A. R. (2008). Sleep disturbance in heavy marijuana users. Sleep (31), 901-908.

Bracken, B. K., Penetar, D. M., Rodolico, J., Ryan, E. T. \& Scott L. E. (2011). Eight weeks of citicoline treatment does not perturb sleep/wake cycles in cocainedependent adults. Pharmacology Biochemistry and Behavior 98(4), 518-524.

Brady T. K. (1998). Comorbitity of substance use and axis I psychiatric disorders. Psychiatry and Mental Health Journal Medscape, 3(4), 1-8.

Castro-Manglano, P. \& Sarrais, O. F. (2011). Protocolo diagnóstico y terapéutico del insomnio. Medicine, 10, 5772-5776.

Coffey, S., Dansky, B. S., Carrigan, M. H. \& Brady, K. T. (2000). Acute and protracted cocaine abstinence in an outpatient population: A prospective study of mood, sleep and withdrawal symptoms. Drug and Alcohol Dependence, 59, 277-286.

Cottler, L. B, Shillington, A. M., Compton, W. M., Mager, D. \& Spitznagel, E. L. (1993). Subjective reports of withdrawal among cocaine users: recommendations for DSM-IV. Drug and Alcohol Dependence, 33, 97-104.

Drake, R. \& Wallach, M. A. (2000). Dual diagnosis: 15 years of progress. Psychiatric Services, 51(9), 11261129.

De la Calzada, D. (2007). Otras hipersomnias. 3r Grup Interdisciplinari de Trastorns del son. Unitat del son Servei des Neurofisiología Hospital Vall Hebrón Barcelona. Acedemia de Ciences Mediques de Catalunya i Balears.
Esqueda, L. \& Velázquez, M. (2011). Fisiología del sueño. En Compendio de fisiología y medicina del sueño (pp.16-27). Grapondi de México: México.

Ferrando, L., Bobes-García, J., Soto, L. F. \& Gilbert, J. (1998). Traducción de la entrevista neuropsiquiátrica MINI, adaptada. Journal of Clinical Psychiatry, 59 (20), 22-23.

Gállego P-L. J., Toledo, J. B., Iriarte, J. \& Urrestarazu, E. (2007). Clasificación de los trastornos del sueño. Anales del Sistema Sanitario de Navarra, 30(1), 19-36. Gillin, J. C., Pulvirenti, L., Withers, N., Golshan, Sh. \& Koob, G. (1994). The effects of lisuride on mood and sleep during acute withdrawal in stimulant abusers: a preliminary report. Biological Psychiatry, 35(11) 843849.

Hauri, P. (1979). What can insomniacs teach us about the function of sleep? En Drucker-Colin, R., Shkurovick, M. \& Sterman, M. B. (Eds.), The functions of sleep. 314 pp. New York: Academic Press.

Hyde, M., Roehrs, T. \& Roth, T. (2005). Alcohol, alcoholism, and sleep. En Lee-Chiong T., Wiley J. \& Sons (Eds.), Sleep: A Comprehensive Handbook (pp. 86787). Hoboken: New Jersey.

Iber, C., Ancoli-Israel, S., Chesson, A. \& Quan, S.F. (2007). The AASM Manual for the Scoring and Associated Events: Rules, Terminology and technical specifications. Westchester: American Academy of Sleep Medicine.

Johanson, C. E., Roehrs, T. \& Schuh, K. (1999). The effects of cocaine on mood and sleep in cocainedependent males. Experimental and Clinical Psychopharmacology, 7, 338-46.

Kovalzon, M. (1990). Las funciones del sueño. En BuelaCasal, G. \& Navarro J. F. (Eds.), Avances en la investigación del sueño y sus trastornos (pp. 3-11). Madrid: Siglo XxI.

Kowatch, R. A., Schnoll, S. S. \& Knisely, J. S. (1992). Electroencephalographic sleep and mood during cocaine withdrawal. Journal of Addictive Disorders, 11(4), 21-45.

Llopis L. J. (2001). Dependencia, intoxicación aguda y síndrome de abstinencia por cocaína. Adicciones, 13(2) 147-165.

Martínez, V. \& Rojas, G. (1999). Técnicas de electroencefalografía. México: Comunicaciones Científicas Mexicanas. 
Matuskey, D., Pittman, B., Forselius, E., Malison, R. T. \& Morgan, P. T. (2011). A multistudy analysis of the effects of early cocaine abstinence on sleep. Drug and Alcohol Dependence, 115, 62-66.

Meana, J. J. \& Barturen, F. (1993). Psicoestimulantes: cocaína, anfetaminas y xantinas. Leioa: Departamento de Farmacología-Universidad del País Vasco.

Medina-Mora, M. E. (2008). Encuesta Nacional de Adicciones (ENA). México: Instituto Nacional de Psiquiatría, Comisión Nacional Contra las Adicciones, CONADIC, SSA.

Miró, E., Martínez, P. \& Arriaza, R. (2006). Influencia de la cantidad y la calidad subjetiva de sueño en la ansiedad y el estado de ánimo deprimido. Salud Mental, 29(2), 30-36.

Morgan, P. T., Pace-Schott, E. F., Sahul, Z. H., Coric, V., Stickgold, R. \& Malison, R. T. (2006). Sleep, sleepdependent procedural learning and vigilance in chronic cocaine users: evidence for occult insomnia. Drug and Alcohol Dependence, 82, 238-249.

Morgan, P. T. \& Malison, R. T. (2007). Cocaine and sleep: early abstinence. The Scientific World Journal, 7(S2), 223-230.

Morgan, P. T., Paliwal, P., Malison, R. T. \& Sinha, R. (2009). Sex differences in sleep and sleep-dependent learning in abstinent cocaine users. Pharmacology Biochemistry and Behavior, 93, 54-58.

Pace-Schott, E., Stickgold, R., Muzur, A., Wigren, P., Ward, A. S. \& Hart, C. (2005). Sleep quality deteriorates over a binge-abstinence cycle in chronic smoked cocaine users. Psychopharmacology, 179, 873-883.

Pace-Schott, E., Stickgold, R., Muzur, A., Wigren, P., Ward, A., Hart, C., Walker, M., Hobson, C.E. \& Hobson, J.A. (2005). Cognitive performance by humans during a smoked cocaine binge-abstinence cycle. The American Journal of Drug and Alcohol Abuse, 31, 571-591.

Pollak, C. P., Thorpy, M. J. \& Yager, J. (1999). The Encyclopedia of Sleep and Sleep Disorders. New York: Infobase Publishing.
Poling, J., Kosten, T. R. \& Sofuoglu, M. (2007). Treatment outcome predictors for cocaine dependence. American Journal of Drug and Alcohol Abuse, 33(2), 191-206.

Prospéro, G., Guzmán, K., Méndez, D., Herrera, S. \& Ruiz, C. (2009). Genes del sueño. Revista de Neurología, 48(4), 199-206.

Satel, S., Price, L., Palumbo, J., McDougle, C., Krystal, J., Gawin, F., Charney, D., Henninger, G. \& Kleber, H. (1991). Clinical phenomenology and neurobiology of cocaine abstinence: a prospective inpatient study. American Journal of Psychiatry, 148, 1712-1716.

spss 19 Para Windows (Software de cómputo). (2010). Chicago: spss, Inc.

Teichner, G., Horner, M. D., Roitzsch, J. C., Herron, J. \& Thevos, A. (2002). Substance abuse treatment outcomes for cognitively impaired and intact outpatients. Addictive Behaviors, 27, 751-763.

Terán, G. (mayo-junio de 2011). Hipersomnias de origen central: diagnóstico y tratamiento. En Diplomado en Polisomnografía: México.

Thompson, P. M., Gillin, J. C., Irwin, M. \& Golshan, Sh. (1995). Polygraphic sleep measures differentiate alcoholics and stimulant abusers during short-term abstinence. Biological Psychiatry, 38 (12), 831-836.

Walsh, S. L., Stoops, W. W., Moody, D. E., Shen-Nan Lin \& Bigelow G. E. (2009). Repeated dosing with oral cocaine in humans: assessment of directs effects, withdrawal, and pharmacokinetics. Experimental and Clinical Psychopharmacology, 17(4) 205-216.

Weddington, W., Brown, B., Haertzen, C., Cone, E., Dax, E., Herning, R. \& Michaelson, B. (1990). Changes in mood, craving, and sleep during short-term abstinence reported by male cocaine addicts: a controlled, residential study. Archives of General Psychiatry, 47, 861-868. 